\title{
Resultados de la cirugía coronaria sin circulación extracorpórea en dos períodos de uso de la técnica
}

\author{
Enrique Seguel ${ }^{1,2}$, Aldo Vera-Calzaretta $^{3}$, Sebastián Ramirez $^{4}$, Roberto González ${ }^{1,2}$, Aleck Stockins $^{1,2}$, Héctor Rubilar. $^{3}$ \\ 1. Universidad de Concepción, Facultad de Medicina, Departamento de Cirugía. \\ 2. Hospital Guillermo Grant Benavente, Centro Cardiovascular. \\ 3. Universidad de Concepción, Facultad de Medicina, Departamento de Salud Pública. \\ 4. Alumno de medicina, Universidad de Concepción.
}

Objetivo: Describir los resultados inmediatos y alejados de la cirugía coronaria sin circulación extracorpórea (CEC) y comparar los resultados de la estrategia de indicación de la técnica en dos períodos.

Pacientes y Métodos: Estudio retrospectivo de 428 pacientes intervenidos de cirugía coronaria sin CEC entre 2004 y 2019 en el Hospital Guillermo Grant Benavente. Se dividen en Grupo 1, período 2004-2008 (N=216) y Grupo 2, período 2009-2019 ( $\mathrm{N}=212)$. Se estudiaron sus características clínicas, resultados quirúrgicos, morbi-mortalidad operatoria y eventos adversos al 31 de junio de 2019 y se compararon los resultados de los grupos a 5 años.

Resultados: No hubo diferencias en la distribución por sexo, edad, factores de riesgo y patologías asociadas entre los grupos. Hubo diferencias en las lesiones coronarias entre los Grupos 1 y 2: lesión de un vaso en $45(20,4 \%)$ versus $125(59 \%)$ y tres vasos en $75(34,5 \%)$ versus $19(9 \%)$ respectivamen- te $(\mathrm{p}<0,001)$. El riesgo operatorio por EuroSCORE logístico fue $3,3 \pm 3,95$ versus $5,4 \pm 7,7$ ( $p<0,001)$. Se confeccionaron 2,3 $\pm 0,9$ anastomosis distales en el Grupo 1 versus $1,3 \pm 0,6$ en Grupo 2 ( $\mathrm{p}<0,001)$. La tasa de complicaciones fueron $17,6 \%$ en el Grupo 1 y $5,7 \%$ en el $2(\mathrm{p}<0,001)$. La mortalidad operatoria globral fue 4 pacientes $(0,9 \%)$. El seguimiento comprendió $9,2 \pm 3,8$ años. La sobrevida a 10 años fue $76,9 \%$ y tasa de eventos cardiovasculares mayores $37,6 \%$. No hubo diferencia entre los grupos a los 5 años.

Conclusiones: La selección de pacientes con anatomía más favorables o de mayor riesgo operatorio tuvo tasas de sobrevida y eventos similares a los observados con la estrategia menos selectiva a 5 años de seguimiento.

Palabras clave: bypass coronario; sin circulación extracorpórea; bypass cardiopulmonar; cirugía cardíaca. 


\section{Off Pump Coronary Artery Surgery : comparison of two consecutive periods with the technique}

Aim: To describe the immediate and long-term results of off pump coronary artery surgery without cardiopulmonary bypass (OPCABG) comparing the results observed in two consecutive periods.

Patients and Methods: A retrospective study of 428 patients undergoing OPCABG between 2004 and 2019 at the Guillermo Grant Benavente Hospital. Group 1 patients operated between 2004 - 2008 $(\mathrm{N}=216)$ and Group 2 operated between 2009 and $2019(\mathrm{~N}=212)$.

Results: There were no differences in sex, age, risk factors and co morbidities between groups. There were differences in the number of coronary lesions between groups: one vessel disease in 45 (20.4\%) versus $125(59 \%)$ and three vessels in $75(34.5 \%)$ versus 19 (9\%) in Group 1 vs Group 2, respectively
( $\mathrm{p}<0.001$ ). The operative risk for logistic EuroSCORE was $3.3 \pm 3.95$ in Group 1 versus $5.4 \pm 7.7$ in Group 2 ( $\mathrm{p}<0.001) .2 .3 \pm 0.9$ distal anastomoses were performed in Group 1 compared to $1.3 \pm 0.6$ in Group 2 ( $\mathrm{p}<0.001)$. Adverse events occurred in $17.6 \%$ o patients in Group 1 compared to $5.7 \%$ in Group 2 ( $\mathrm{p}<0.001)$. Overall, 4 patients died $(0.9 \%)$. Mean overall survival at 10 years was $76.9 \%$ and the rate of major cardiovascular events was $37.6 \%$, no differences being observed between groups.

Conclusions: At 5 years of follow-up the selection of patients with more favorable anatomy or greater operative risk had similar survival rates and events than those observed with the least selective strategy. Key words: coronary artery bypass; off pump; cardiopulmonary bypass; cardiac surgical procedures. 


\section{Introducción:}

El tratamiento quirúrgico estándar para la enfermedad coronaria es la cirugía de revascularización miocárdica con circulación extracorpórea (CEC). Esta técnica permite la realización de anastomosis coronarias en un campo exangüe, con un corazón inmóvil. A pesar de que actualmente la CEC es una técnica segura para los pacientes, se asocia a algunas complicaciones como activación de una respuesta inflamatoria, accidentes cerebrovasculares, disfunción plaquetaria y otras. ${ }^{1}$

Durante los años 70 se desarrollaron técnicas de revascularización sin el uso de CEC. Mediante el uso de posicionadores cardiacos y estabilizadores coronarios, se logra inmovilizar el segmento del corazón donde se realizan la anastomosis y éstas pueden ser confeccionadas con una adecuada visualización y seguridad. ${ }^{2-4}$ Los estudios iniciales sobre esta técnica mostraron que era tan segura como la cirugía con bomba, con beneficios adicionales como la reducción de daño miocárdico y cerebral, la tasa de transfusiones, el costo y la duración de la estadía hospitalaria. Varias bases de datos que reflejaban la experiencia del "mundo real" informaron una reducción de la mortalidad y la morbilidad, con mayor impacto en pacientes de mayor riesgo. 5

El registro del año 2009 de la Sociedad de Cirujanos Torácicos (STS) en Estados Unidos mostró que un $21 \%$ de las cirugías coronarias se realizaron sin CEC. ${ }^{6}$

El $4^{\circ}$ Reporte de la Base de Datos de la Asociación Europea de Cirugía Cardio Torácica del 2010 mostró que un $21 \%$ de ellas se realizó sin CEC. ${ }^{7}$

En nuestro centro iniciamos la técnica el año 2004 y el año 2007 realizamos casi el 30\% de nuestras cirugías coronarias sin CEC.

Sin embargo, los resultados de dos grandes estudios randomizados, ROBBY y CORONARY no mostraron diferencias en la mortalidad operatoria, tasas de infarto perioperatorio, accidente cerebrovascular y falla renal aguda. En cambio, se observó la realización de un menor número de puentes, una menor permeabilidad de los mismos y una mayor necesidad de revascularización repetida en el grupo operado sin CEC, cuestionando la calidad técnica de las anastomosis realizadas con la técnica. ${ }^{8-10}$

En años posteriores el entusiasmo por la técnica disminuyó y solo se mantuvo en algunos centros de alto volumen o se reservó, como en nuestro centro, para pacientes seleccionados.

El objetivo de esta comunicación es describir los resultados inmediatos y alejados de la cohorte de pacientes operados con cirugía coronaria sin CEC desde el inicio de la técnica en nuestro hospital y comparar los resultados observados en dos períodos en los que se usaron dos estrategias en la indicación de la técnica.

\section{Método:}

Estudio descriptivo de los resultados operatorios, sobrevida y eventos cardiovasculares mayores de los pacientes intervenidos de cirugía coronaria sin CEC entre 2004 y 2019 en el Hospital Guillermo Grant Benavente $(\mathrm{N}=428)$.

Los datos fueron extraídos del registro de cirugías cardíacas del centro cardiovascular, la base de datos del hospital y los registros del laboratorio de hemodinamia, construyéndose una base de datos anonimizada.

\section{Pacientes:}

Grupo 1: Pacientes intervenidos entre 2004 y 2008, período en que se seleccionaron pacientes considerando la factibilidad técnica y a la experiencia del grupo. La serie se inició con pacientes con enfermedad de un vaso (descendente anterior) y progresivamente se avanzó a pacientes con enfermedad coronaria de los otros territorios $(\mathrm{N}=216)$.

Grupo 2: Pacientes intervenidos entre el 2009 y 2019 , cuando la técnica se reservó para pacientes con enfermedad coronaria menos extensa o pacientes con un riesgo mayor (ateromatosis aórtica, daño renal, enfermedad pulmonar) en los que se quería evitar la CEC $(\mathrm{N}=212)$.

\section{Técnica quirúrgica}

El detalle de la técnica quirúrgica puede encontrarse en la publicación previa de nuestro grupo. ${ }^{11}$

\section{Variables}

Se tabularon las características clínicas y demográficas, el riesgo operatorio calculado por EuroSCORE aditivo y logístico ${ }^{12,13}$, la extensión de la enfermedad coronaria, el número y objetivos de las anastomosis distales. Las complicaciones post operatorias se definieron como: quirúrgicas (hemorragia, reoperación), neurológicas (accidente vascular encefálico), renales (insuficiencia renal aguda con o sin necesidad de diálisis), cardíacas (arritmias, infarto perioperatorio, shock) e infecciones (herida operatoria, mediastinitis e infección urinaria).

La mortalidad operatoria se consideró hasta los 30 días 
post cirugía o hasta el alta hospitalaria si el paciente permaneció más de un mes hospitalizado.

\section{Seguimiento}

El seguimiento se completó hasta el 31 de junio de 2019.

La sobrevida y causas de muerte se obtuvieron de acuerdo con los datos del Servicio de Registro Civil e Identificación.

El seguimiento de eventos clínicos se hizo de acuerdo con el registro clínico del centro cardiovascular.

Se estudiaron la mortalidad alejada, identificando aquella de causa cardiovascular y no cardiovascular; y los eventos cardiovasculares mayores: infarto agudo al miocardio (IAM), accidente cerebro vascular (AVE) y reintervención, así como también la recurrencia de la angina.

\section{Análisis Estadístico}

Se realizó análisis descriptivo univariado utilizando gráficos y tablas para describir las distribuciones porcentuales de variables nominales u ordinales. Se utilizaron medidas de tendencia central y de dispersión para las variables cuantitativas.

Se utilizó el método Kaplan Meier para el estudio de la supervivencia global, de causas cardíacas y otras causas. Debido a que los períodos de seguimiento de los dos grupos son diferentes, se compararon los resultados alejados a los 5 años.

Para el procesamiento estadístico se utilizaron los softwares estadísticos IBM SPSS 21.0 y el Stata 14.

\section{Resultados:}

Las cirugías sin CEC correspondieron al 11,4\% de las 3.763 cirugías coronarias exclusivas realizadas en el período. Su uso tuvo un máximo entre los años 20062008, en que representó el 29,2\% de las cirugías coronarias; y un mínimo el 2010, en que sólo un 2,2\% de las cirugías se hicieron sin CEC. Desde el 2013 hasta hoy representa el 5,2\% de las cirugías coronarias.

\section{Pacientes}

Corresponden a 322 hombres $(75,2 \%)$ con una edad media $61,8 \pm 10$ años.

Los factores de riesgo cardiovascular y patologías asociadas se describen en la Tabla 1. No hubo diferencias en la distribución por género, edad, factores de riesgo y patologías asociadas de los pacientes entre los grupos. El promedio de vasos enfermos de la serie fue de 1,97.

\section{Tabla 1: Pacientes}

\begin{tabular}{|lll|ll|ll|l|}
\hline & \multicolumn{1}{l|}{ Total } & $\mathbf{2 0 0 4 - 2 0 0 8}$ & $\mathbf{2 0 0 9 - 2 0 1 9}$ & \\
& $\mathrm{n}=428$ & $\%$ & $\mathrm{n}=216$ & $\%$ & $\mathrm{n}=212$ & $\%$ & $\mathrm{p}$ \\
SEXO & & & & & & & \\
Hombre & 322 & 75,2 & 165 & 76,4 & 157 & 74,1 & 0,576 \\
Mujer & 106 & 24,8 & 51 & 23,6 & 55 & 25,9 & \\
EDAD & 61,8 & $\pm 10,1$ & 60,8 & $\pm 8,9$ & 62,9 & $\pm 11,2$ & 0,006 \\
FACTORES DE RIESGO & & & & & & & \\
Sobrepeso & 133 & 31,1 & 94 & 43,5 & 39 & 18,4 & 0,614 \\
Obesidad & 96 & 22,4 & 64 & 29,6 & 32 & 15,1 & 0,001 \\
Hipertensión Arterial & 319 & 74,5 & 182 & 84,3 & 137 & 64,6 & 0,563 \\
Diabetes Mellitus & 126 & 29,4 & 64 & 29,6 & 62 & 29,2 & 0,102 \\
Dislipidemia & 146 & 34,1 & 92 & 42,6 & 54 & 25,5 & 0,050 \\
Tabaquismo & 152 & 35,5 & 95 & 44 & 57 & 26,9 & 0,062 \\
PATOLOGIAS ASOCIADAS & & & & & & & \\
EPOC & 17 & 4 & 10 & 4,6 & 7 & 3,3 & 0,033 \\
Enfermedad Renal Crónica & 26 & 6,1 & 10 & 4,6 & 16 & 7,5 & 0,052 \\
AVE previo & 13 & 3 & 5 & 2,3 & 8 & 3,8 & 0,177 \\
\hline
\end{tabular}

Tabla 2: Condición cardiovascular pre operatoria.

\begin{tabular}{|c|c|c|c|c|c|c|c|}
\hline & \multicolumn{2}{|l|}{ Total } & \multicolumn{2}{|c|}{ 2004-2008 } & \multicolumn{2}{|c|}{ 2009-2019 } & \multirow[b]{2}{*}{$p$} \\
\hline & $n=428$ & $\%$ & $n=21$ & $\%$ & $n=212$ & $\%$ & \\
\hline \multicolumn{8}{|c|}{ LESIONES CORONARIAS } \\
\hline Lesión TCl & 61 & 14,3 & 36 & 16,7 & 25 & 11,8 & 0,506 \\
\hline Lesión 1 vaso & 170 & 39,7 & 45 & 20,8 & 125 & 59 & 0,001 \\
\hline Lesión 2 vasos & 103 & 24,1 & 60 & 27,8 & 43 & 20,3 & 0,728 \\
\hline Lesión 3 vasos & 94 & 22 & 75 & 34,7 & 19 & 9 & 0,001 \\
\hline \multicolumn{8}{|l|}{ CONDICIÓN } \\
\hline \multicolumn{8}{|l|}{ CARDIOVASCULAR } \\
\hline Angina Estable & 261 & 61 & 150 & 69,4 & 111 & 52,4 & 0,584 \\
\hline Angina inestable & 93 & 21,7 & 65 & 30,1 & 28 & 13,2 & 0,009 \\
\hline Infarto < 90días & 108 & 25,2 & 56 & 25,9 & 52 & 24,5 & 0,216 \\
\hline $\mathrm{PCl}$ previa & 84 & 19,6 & 48 & 22,2 & 36 & 17 & 0,925 \\
\hline \multirow{2}{*}{\multicolumn{8}{|c|}{$\begin{array}{l}\text { FRACCIÓN EYECCIÓN VI } \\
\text { (rango } 25-80 \% \text { ) }\end{array}$}} \\
\hline & & & & & & & \\
\hline$>50 \%$ & 323 & 75,5 & 151 & 69,9 & 172 & 81,1 & 0,276 \\
\hline $30-50 \%$ & 91 & 21,3 & 56 & 25,9 & 35 & 16,5 & 0,365 \\
\hline$<30 \%$ & 14 & 3,3 & 9 & 4,2 & 5 & 2,4 & 0,598 \\
\hline \multicolumn{8}{|c|}{ RIESGO OPERATORIO } \\
\hline EuroSCORE Aditivo & 3,5 & $\pm 2,83$ & 3,2 & $\pm 2,57$ & 4,3 & $\pm 3,25$ & 0,016 \\
\hline EuroSCORE log & 3,9 & $\pm 5,43$ & 3,3 & $\pm 3,95$ & 5,4 & $\pm 7,7$ & 0,001 \\
\hline
\end{tabular}

TCI: Tronco coronario izquierdo

PCI: Angioplastía coronaria percutánea.

VI: Ventrículo izquierdo. 


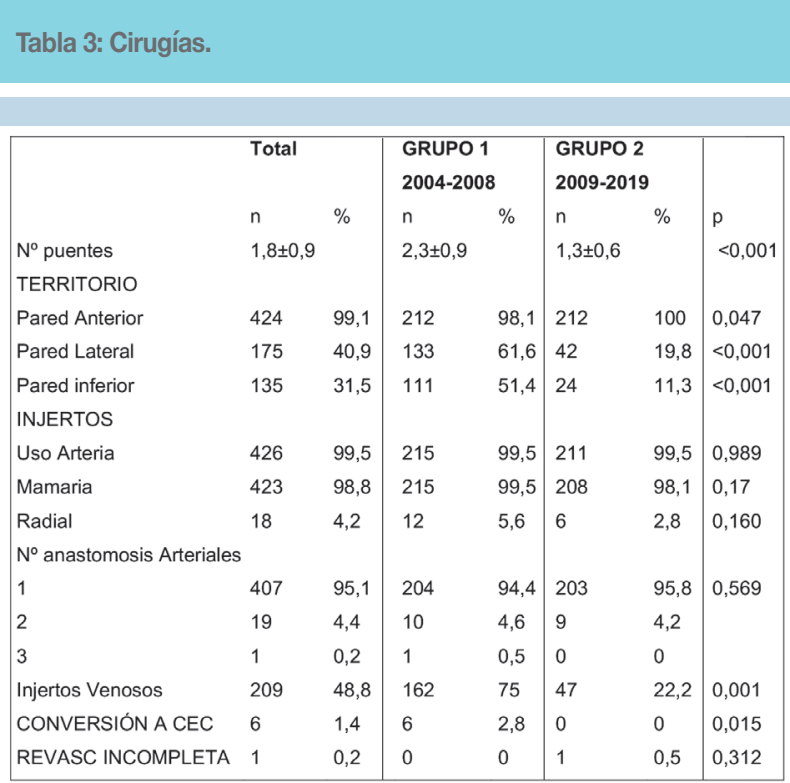

REVASC: Revascularización.

Estadístico Chi - cuadrado es significativo con un valor de 0,05.

\section{Tabla 4: Complicaciones post operatorias.}

\begin{tabular}{|c|c|c|c|c|c|c|c|}
\hline & \multicolumn{2}{|l|}{ Total } & \multicolumn{2}{|c|}{$\begin{array}{l}\text { GRUPO } 1 \\
2004-2008\end{array}$} & \multicolumn{2}{|c|}{$\begin{array}{l}\text { GRUPO } 2 \\
2009-2019\end{array}$} & \multirow{4}{*}{$\begin{array}{l}p \\
<0,001\end{array}$} \\
\hline & $n$ & $\%$ & $\mathrm{n}$ & $\%$ & $\mathrm{n}$ & $\%$ & \\
\hline $\mathrm{N}^{\circ}$ puentes & \multicolumn{2}{|c|}{$1,8 \pm 0,9$} & \multicolumn{2}{|c|}{$2,3 \pm 0,9$} & \multicolumn{2}{|c|}{$1,3 \pm 0,6$} & \\
\hline \multicolumn{7}{|l|}{ TERRITORIO } & \\
\hline Pared Anterior & 424 & 99,1 & 212 & 98,1 & 212 & 100 & 0,047 \\
\hline Pared Lateral & 175 & 40,9 & 133 & 61,6 & 42 & 19,8 & $<0,001$ \\
\hline Pared inferior & 135 & 31,5 & 111 & 51,4 & 24 & 11,3 & $<0,001$ \\
\hline \multicolumn{8}{|l|}{ INJERTOS } \\
\hline Uso Arteria & 426 & 99,5 & 215 & 99,5 & 211 & 99,5 & 0,989 \\
\hline Mamaria & 423 & 98,8 & 215 & 99,5 & 208 & 98,1 & 0,17 \\
\hline Radial & 18 & 4,2 & 12 & 5,6 & 6 & 2,8 & 0,160 \\
\hline \multicolumn{8}{|l|}{$\mathrm{N}^{\circ}$ anastomosis Arteriales } \\
\hline 1 & 407 & 95,1 & 204 & 94,4 & 203 & 95,8 & 0,569 \\
\hline 2 & 19 & 4,4 & 10 & 4,6 & 9 & 4,2 & \\
\hline 3 & 1 & 0,2 & 1 & 0,5 & 0 & 0 & \\
\hline Injertos Venosos & 209 & 48,8 & 162 & 75 & 47 & 22,2 & 0,001 \\
\hline CONVERSIÓN A CEC & 6 & 1,4 & 6 & 2,8 & 0 & 0 & 0,015 \\
\hline REVASC INCOMPLETA & 1 & 0,2 & 0 & 0 & 1 & 0,5 & 0,312 \\
\hline
\end{tabular}

IAM: Infarto agudo al miocardio.

AVE: Accidente vascular encefálico.

*Estadístico Chi-cuadrado es significativo con un valor de 0,05.
Tabla 5: Eventos en el seguimiento a 9 años.

\begin{tabular}{|lll|}
\hline & $\mathrm{n}$ & $\%$ \\
Fallecido & 99 & 23,1 \\
Fallecen Causa CV & 49 & 11,8 \\
IAM & 35 & 10,2 \\
AVE & 27 & 7,9 \\
Re intervención & 29 & 8,5 \\
MACCE & 140 & 38,4 \\
Recurrencia Angina & 73 & 17,1 \\
\hline
\end{tabular}

CV: Cardio vascular

IAM: Infarto agudo al miocardio

AVE: Accidente vascular encefálico.

MACCE: Eventos cardiovasculares y cerebrovasculares mayores.

Hubo diferencias en las lesiones coronarias entre los grupos: lesión de un vaso en $45(20,4 \%)$ del Grupo 1 y $125(59 \%)$ del Grupo 2 ( $\mathrm{p}<0,001)$; y lesión de tres vasos en $75(34,5 \%)$ del Grupo 1 y 19 (9\%) del Grupo $2(\mathrm{p}<0,001)$.

El Grupo 2 tuvo un riesgo operatorio calculado por EuroSCORE logístico mayor que el Grupo 1 (5,4 7,7 versus $3,3 \pm 3,95 ; \mathrm{p}<0,001)$. (Tabla 2).

\section{Cirugías}

Se confeccionaron un promedio $1,8 \pm 0,9$ puentes/paciente.

En el Grupo 1 se confeccionaron más anastomosis

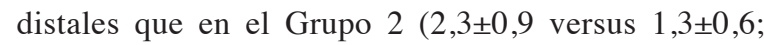
$\mathrm{p}<0,001)$ y hubo más pacientes a los que se realizaron anastomosis a la pared lateral (61,6 versus $19,8 \%$, $\mathrm{p}<0,001)$ e inferior $(51,4$ versus $11,3 \%$, respectivamente, $\mathrm{p}<0,001)$.

Se utilizaron injertos arteriales en el 99,5\% de los pacientes, sin diferencias entre los grupos, pero hubo un mayor uso de injertos venosos en el Grupo 1 ( 75 versus $22,2 \%, \mathrm{p}<0,001)$.

Hubo 6 conversiones a cirugía con CEC $(1,4 \%)$, todos en el Grupo 1, y en un caso no se hicieron todos los puentes planeados, lo que se consideró revascularización incompleta (en el Grupo 2).

Los resultados quirúrgicos se muestran en la Tabla 3. 
Figura 1: Sobrevida global

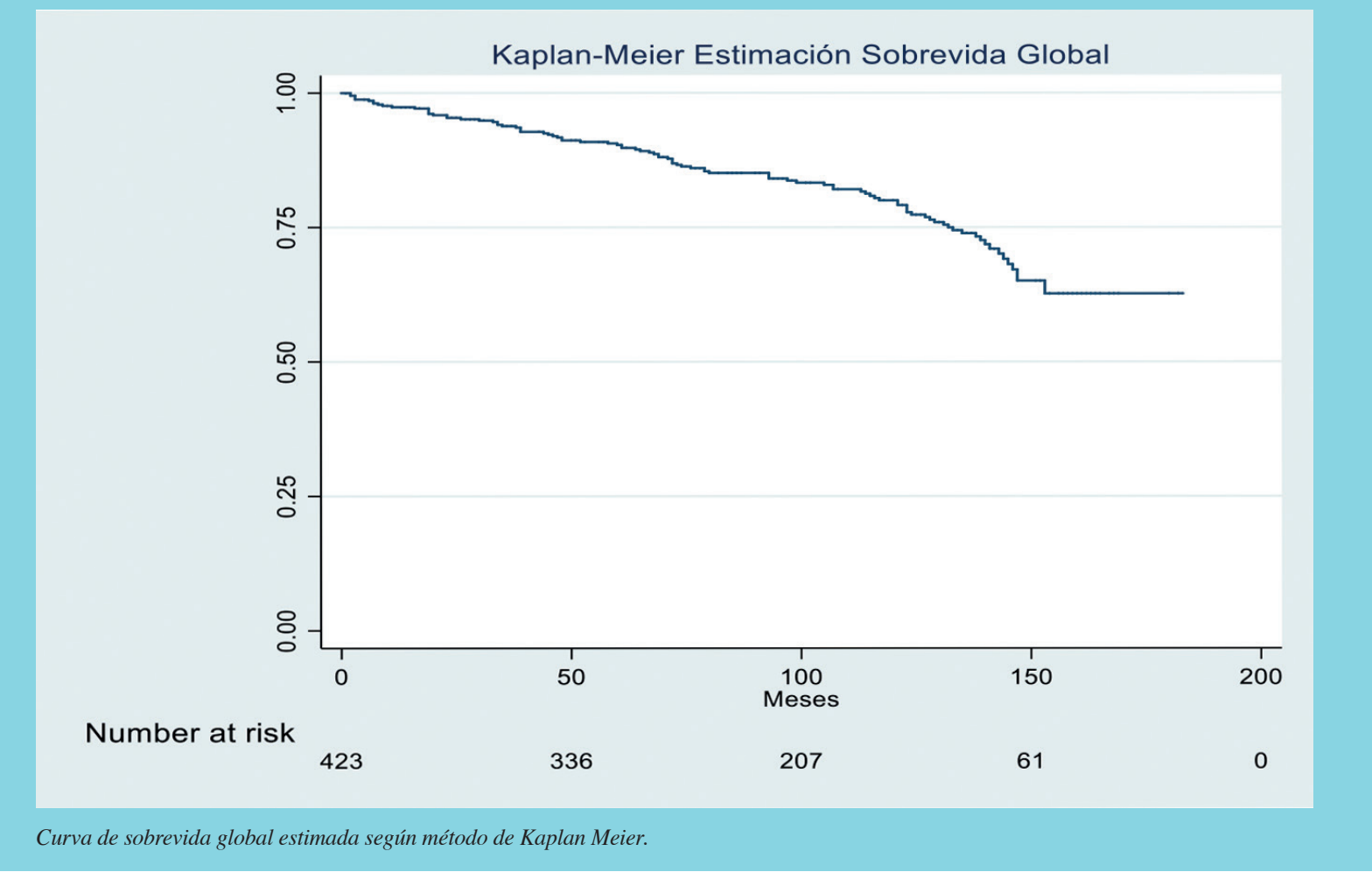

Figura 2: Curva de sobrevida libre de muerte de causa cardiovascular según método de Kaplan Meier.

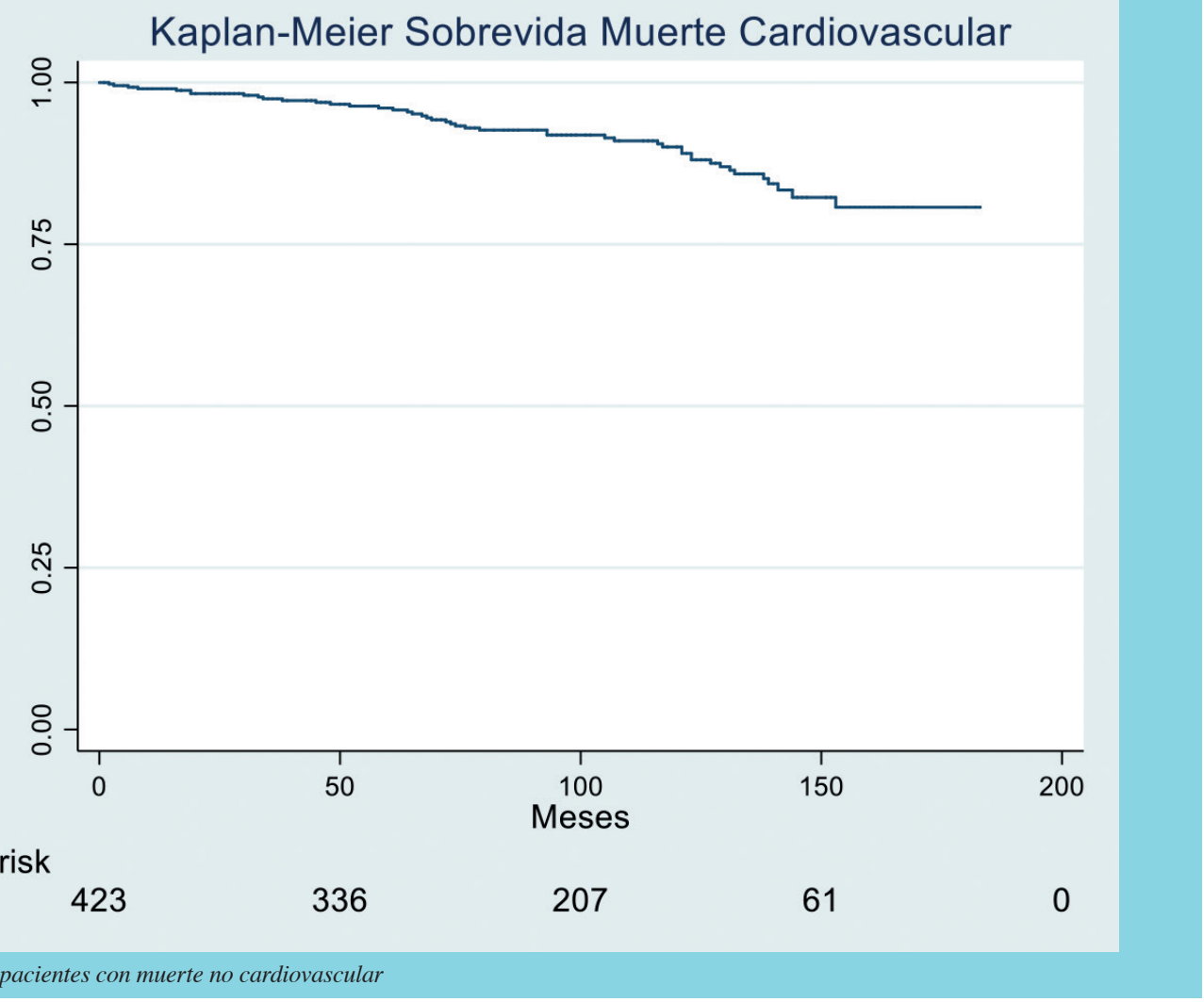



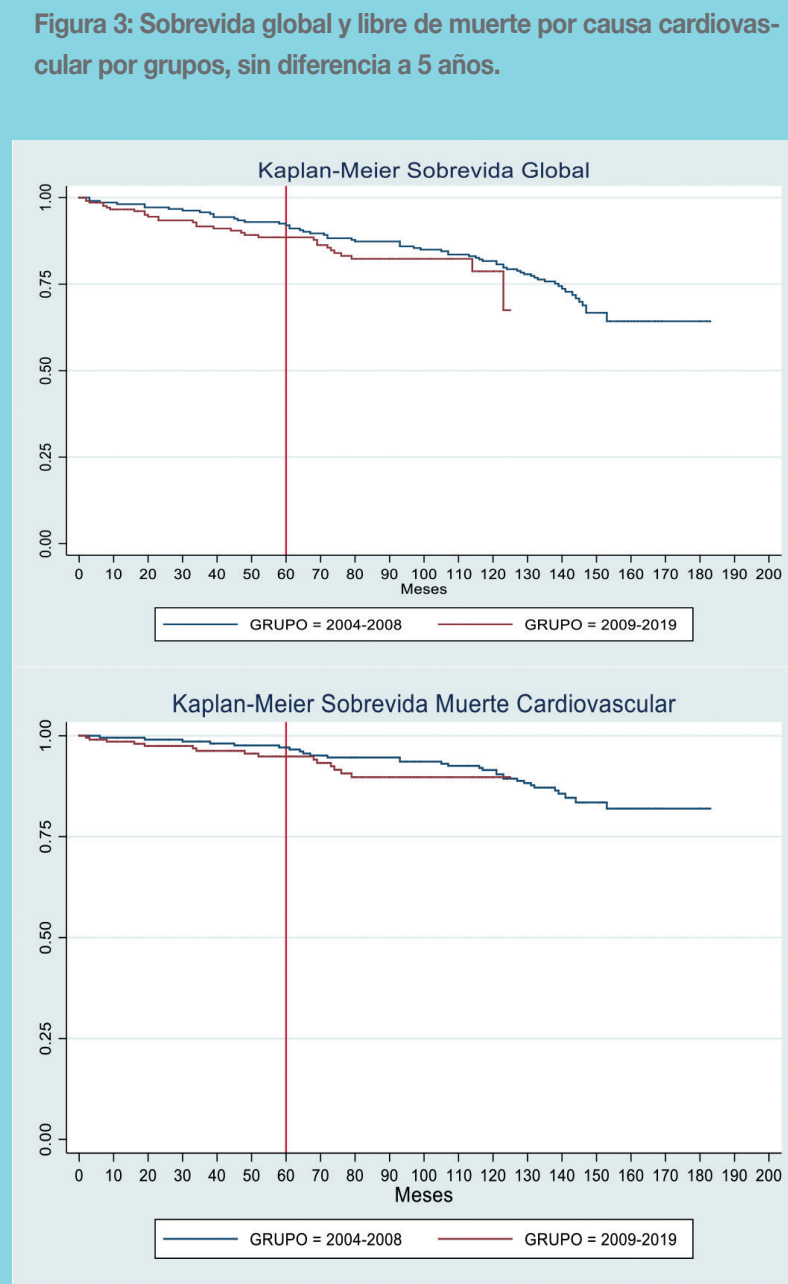

\section{Complicaciones}

Hubo una mayor necesidad de transfusiones en el grupo $1(13 \%$ versus $2,8 \%$; $<<0,001)$.Se presentaron complicaciones en el $11,7 \%$ de los pacientes.

Hubo menos complicaciones totales en el Grupo 2 (5,7 versus $17,6 \% ; \mathrm{p}<0,001)$; sin embargo, no hubo diferencias significativas en las complicaciones específicas médicas o quirúrgicas.

Fallecieron 4 pacientes (0,9\%), 3 en el Grupo 1 y 1 en el Grupo 2. Las causas fueron muerte súbita en dos casos (bradicardia en uno y fibrilación ventricular en otro), síndrome urémico y shock refractario post hemorragia post operatoria.

El detalle de las complicaciones y mortalidad operatoria se presenta en la Tabla 4.

\section{Seguimiento}

El seguimiento de la serie fue de $9,2 \pm 3,8$ años. Para el Grupo 1 fue de 12,25 y el Grupo 2 fue 6,1 años. Por problemas en el registro del Rut de 13 pacientes, el seguimiento se la sobrevida se logró en 415 pacientes (97\%). Fallecieron 99 pacientes y la sobrevida a 10 años fue de $76,9 \%$.

Las causas de muerte fueron cardiovasculares en 49 pacientes: paro cardio respiratorio en 18 , infarto agudo al miocardio en 14, insuficiencia cardiaca en 9 y accidente cerebro vascular en 8 . La sobrevida libre de muerte de causa cardiovascular fue $87 \%$ (Figuras 1 y 2 ).

No hubo diferencias en la sobrevida global ni en la supervivencia libre de mortalidad de causa cardiovascular entre los grupos a 5 años (Figura 3).

El seguimiento de eventos clínicos se logró en 342 pacientes $(79,9 \%)$. Los otros pacientes no tienen control clínico en nuestro centro y lo hacen en sus hospitales de referencia.

Hubo reintervenciones en 29 pacientes: consistieron en 25 coronariografías (a 15 de ellos se realizó una angioplastía: 4 a la descendente anterior y 11 a otro vaso) y hubo 4 re operaciones. Se instalaron además 3 marcapasos y 2 desfibriladores.

Los eventos cardiovasculares y cerebrovasculares mayores de la serie se describen en la Tabla 5.

La tasa de eventos cardiovasculares y cerebrovasculares mayores (MACCE) a 5 años fue similar entre los grupos y no hubo diferencias en la sobrevida libre de infartos, accidentes cerebrovasculares, ni reintervenciones (Figuras 4 y 5 ).

\section{Discusión:}

La cirugía sin CEC es una técnica alternativa a la cirugía coronaria tradicional que busca evitar las complicaciones asociadas a la CEC. ${ }^{14,15} \mathrm{Su}$ seguridad fue demostrada en distintos estudios que reportaron sus ventajas, especialmente en pacientes de alto riesgo quirúrgico, diabéticos y mayores de 80 años. ${ }^{16-18} \mathrm{Su}$ adopción por diferentes centros fue progresivamente en aumento y hoy en día representa el $60 \%$ de las cirugías coronarias en Asia y el 15-20\% en el mundo occidental. ${ }^{19}$ - 21

Iniciamos su uso el año 2004, adquiriendo una experiencia progresiva hasta llegar a realizar más de un $25 \%$ de nuestras cirugías coronarias con ella entre los años 2006 y 2008. Se intervinieron progresivamente pacientes con lesión de uno, dos y tres vasos, así como pacientes con lesiones de tronco coronario izquierdo. 


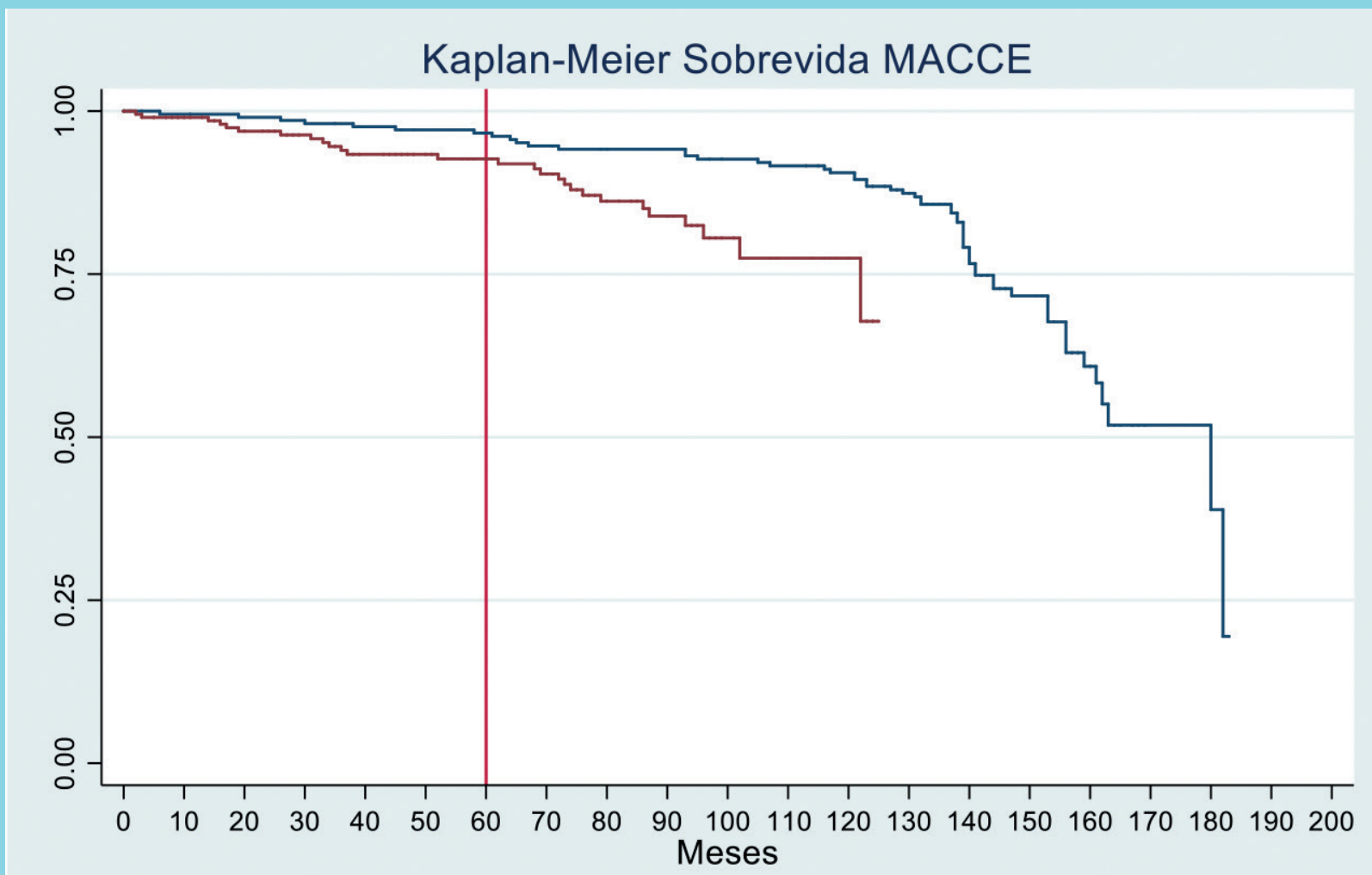

GRUPO = 2004-2008

GRUPO = 2009-2019

Sobrevida excluyendo pacientes con muerte no cardiovascular

En general correspondieron a pacientes de bajo riesgo quirúrgico. Los resultados de ese período fueron comparables a la cirugía con CEC. ${ }^{22}-23$

A pesar se lo extendido de su uso, la controversia sobre sus beneficios continúa abierta. Los resultados reportados en los primeros estudios observacionales no han sido consistentes con los obtenidos en estudios randomizados y metaanálisis publicados posteriormente. ${ }^{24}$ En el estudio ROOBY, realizado en los Hospitales de Veteranos en Estados Unidos, la mortalidad a 30 días fue $2 \%$ y $1 \%$ para la cirugía con y $\sin$ CEC $(p=0,47)$. La cirugía sin CEC se asoció a más pacientes en quienes se realizaron menos puentes que los planeados $(17,8 \%$ vs $11,1 \%, \mathrm{p}<0,001)$ y el seguimiento angiográfico a doce meses reportó una menor permeabilidad de los puentes $(82,6 \%$ vs $87,8 \%, \mathrm{p}<0,001) .{ }^{8}$
El estudio CORONARY, estudio multicéntrico realizado en 79 centros de 19 países y que incluyó 4.752 pacientes con distintos niveles de riesgo, no mostró diferencias en la mortalidad, IAM, AVE o falla renal a 30 días y a 12 meses. La cirugía sin CEC se asoció a una reducción de las transfusiones, complicaciones respiratorias y reoperaciones por sangrado. ${ }^{9}$ La supervivencia y los eventos clínicos a 5 años fue similar.10 En el estudio GOPCABE, que incluyó solo a pacientes mayores de 75 años, tampoco se mostró diferencias significativas entre ambas técnicas. ${ }^{25}$

Debido a que en nuestra experiencia inicial con la cirugía sin CEC tampoco observamos los beneficios que esperábamos, desde el año 2009 comenzamos a reservarla para pacientes en los que pensábamos que sí habría un: aquellos con enfermedad coronaria limitada a 
Figura 5: Curvas de eventos por grupos: infarto agudo al miocardio (IAM), accidente vascular encefálico, y reintervenciones por grupos. No hubo diferencia a 5 años.
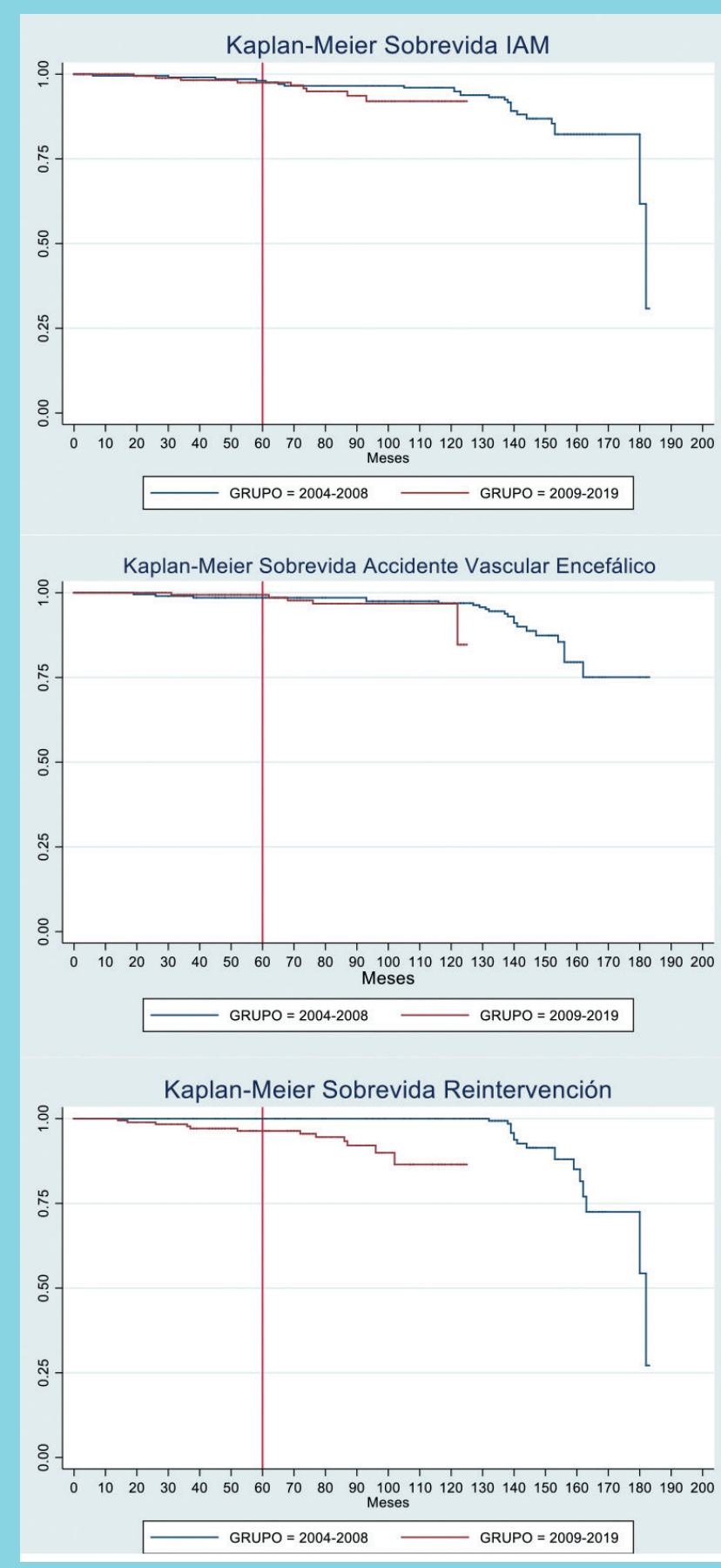

uno o dos vasos, con anatomía favorable, y pacientes con un mayor riesgo quirúrgico.

Debido a esta selección, los pacientes intervenidos en el período 2009-2019 tuvieron una menor extensión de su enfermedad coronaria y un riesgo operatorio mayor. Eso se tradujo en que el número de puentes realizados fue significativamente menor, hubo menos puentes a las paredes lateral e inferior y menos uso de injertos venosos.

Por oro lado, esta estrategia se asoció a una menor tasa de conversión, menor necesidad de transfusiones y de complicaciones postoperatorias globales. No hubo diferencias en las complicaciones específicas ni en la mortalidad operatoria entre los grupos a pesar de que el riesgo operatorio del Grupo 2 era significativamente mayor. La sobrevida global, las tasas de muerte de causa cardiovascular y los eventos a 5 años fueron similares entre los grupos.

En el mediano plazo la tasa de eventos de la serie fue comparable a lo publicado para la cirugía coronaria, con o sin CEC. 26,27

Actualmente, nuestro grupo privilegia la cirugía coronaria con CEC con pinzamiento aórtico único y un mayor uso de injertos arteriales, y la reservamos para pacientes seleccionados con anatomía coronaria favorable y/o mayor riesgo operatorio. En estos pacientes la técnica mostró excelentes resultados, disminuyó la necesidad de trasfusiones, tuvo una baja tasa de complicaciones y mortalidad. En el seguimiento las tasas de sobrevida, infarto y AVE fueron similares a la estrategia menos selectiva.

\section{Limitaciones}

Se trata de una serie retrospectiva, de pacientes seleccionados, que no representan al universo de pacientes con enfermedad coronaria del centro.

Los resultados están influenciados por el sesgo en la selección y la experiencia del equipo quirúrgico.

No se realizó control angiográfico de rutina a los pacientes, por lo que la permeabilidad de los puentes se desconoce.

Debido a que se trata de un centro de referencia, los pacientes provenientes de otros hospitales se controlan en sus centros de origen y el seguimiento de eventos clínicos sólo se completó en un $80 \%$.

Los períodos de seguimiento de los grupos difieren, por lo que el corte a 5 años puede no reflejar la evolución posterior. 


\section{Conclusiones:}

La cirugía coronaria sin CEC tuvo una baja tasa de complicaciones y mortalidad operatorias, una baja tasa de eventos y buena sobrevida a 9 años.

A 5 años de seguimiento, la estrategia de selección de pacientes con anatomía más favorables o de mayor riesgo operatorio del segundo periodo tuvo tasas de sobrevida y eventos similares a los observados con la estrategia menos selectiva del primer período.

\section{Referencias}

1. RAJA S, BERG G. Impact of off pump coronary artery bypass surgery on systemic inflammation: current best available efidence. J Card Surg 2007; 22: 445-55.

2. BENETTI F, NASELLI G, WOOD M, GEFFNER L. Direct myocardial revascularization without extracorporeal circulation. Experience in 700 patients. Chest 1991; 100: 312-6.

3. BUFFOLO E, ANDRADE JC, RODRIGUEZ J, TELES C, FIGUEIREDO L, GOMES W. Direct myocardial revascularization without cardiopulmonary bypass. Thorac Cardiovasc Surg 1985; 33: 26-9.

4. BUFFOLO E, ANDRADE JC, BRANCO JN, AGUIAR LF, RIBEIRO EE, JATENE AD. Myocardial revascularization without extracorporeal circulation. Seven years experience in 593 cases. Eur J Cardiothorac Surg 1990; 4: 504-7.

5. ABU-OMAR Y, TAGGART DP. The present status of offpump coronary artery bypass grafting. Eur J Cardiothorac Surg 2009; 36: 312-21.

6. EL BARDISSI A, ARANKI S, SHENG S, O'BRIEN S. GREENBERG C, GAMMIE J. Trends in isolated coronary artery bypass grafting: An análisis of the Society of Thoracic Surgeons adult cardiac surgery database. J Torac Cardiovas Surg 2012: 143: 273-81.

7. BRIDGEWATER B, KINSMAN R, WALTON P, GUMMERT J, KAPPETEIN AP. The 4th European Association for Cardio-Thoracic Surgery adult cardiac surgery database report. Interact Cardiovasc Thorac Surg 2011; 12: 4-5.

8. SHROYER A, GROVER F, ALTER B, COLLINS J, MCDO-
NALD G, KOZORA E, et al, for the Veterans Affairs Randomized On/Off Bypass (ROOBY) Study Group. On-Pump versus Off-Pump Coronary-Artery Bypass Surgery. N Engl J Med 2009; 361:1027-37.

9. LAMY A, DEVEREAUX J, PRABHAKARAN D, TAGGART D, HU S, PAOLASSO E, ET AL (For The Coronary Investigators). Off-Pump Or On-Pump Coronary-Artery Bypass Grafting At 30 Days. N Engl J Med 2012; 366: 1489-97.

10. LAMY A, DEVEREAUX PJ, PRABHAKARAN D, TAGGART DP, HU S, STRAKA Z, et al. Five year outcomes after off-pump or on-pump coronary-artery bypass grafting. N Engl J Med 2016; 375: 2359-68.

11. GONZÁLEZ R, SEGUEL E, STOCKINS A, CAMPOS R, NEIRA L, ALARCÓN E. Cirugía Coronaria: Revascularización miocárdica sin circulación extracorpórea. Rev Chil Cir 2009; 61: 578-81.

12. NASHEF SA, ROQUES F, MICHEL P, GAUDUCHEAU E, LEMESHOW S, SALAMON R. European system for cardiac operative risk evaluation (EuroSCORE). Eur J Cardiothorac Surg 1999; 16: 9-13.

13. ROQUES F, MICHEL P, GOLDSTONE AR, NASHEF SA. The logistic EuroSCORE. Eur Heart J 2003; 24: 882-3.

14. TRAPP W, BISARYA R. Placement of coronary artery bypass graft without pump-oxigenator. Ann Thorac Surg 1975; 19: 1-9.

15. ANKENEY J. To use or not to use the pump oxigenator in coronary bypass operations. Ann Thorac Surg 1975; 19: 108-9. 
16. CLEVELAND JC JR, SHROYER AL, CHEN AY, PATERSON E, GROVER FL. Off-pump coronary artery bypass grafting decreases risk-adjusted mortality and morbidity. Ann Thorac Surg 2001; 72: 1282-8.

17. BENEDETTO U, CAPUTO M, VOHRA H, DAVIES A, HILLIER J, BRYEAN A, et al. Off-pump versus on-pump coronary artery bypass surgery in patients with actively treateddiabetes and multivessel coronary disease. $\mathrm{J}$ Thorac Cardiovasc Surg 2016; 152: 1321-30.

18. HOFF SJ, BALL SK, COLTHARP WH, GLASSFORD DM JR, LEA JD 4TH, PETRACEK MR. Coronary artery bypass in patients 80 years and over: is off - pump the operation of choice? Ann Thorac Surg. 2002; 74: S1340-3.

19. TAGGART DP, ALTMAN DG. Off-pump vs. on-pump CABG: are we any closer to a resolution? Eur Heart J 2012; 33: 1181-3.

20. HUFFMYER J, RAPHAEL J. The current status of off-pump coronary bypass surgery. Curr Opin Anaesthesiol 2011; 24: 64-9.

21. PATEL NN, ANGELINI GD. Off-pump coronary artery bypass grafting: for the many or the few? J Thorac Cardiovasc Surg 2010; 140: 951-3.

22. SEGUEL E, GONZÁLEZ R, STOCKINS A, ALARCÓN E, CONCHA R. Resultados Inmediatos y a Mediano Plazo de
La Cirugía Coronaria sin Circulación Extracorpórea. Rev Med Chi 2013; 141: 281-90.

23. GONZÁLEZ R. Cirugía coronaria sin circulación extracorpórea: comparación con cirugía de revascularización miocárdica convencional con método Propensity Score Matching. Rev Cir 2019;71:299-306.

24. NATHOE HM, VAN DIJK D, JANSEN EW, SUYKER WJ, DIEPHUIS JC, VAN BOVEN WJ, et al. A comparison of onpump and off pump coronary bypass surgery in low-risk patients. N Engl J Med. 2003; 348: 394-402.

25. DIEGELER A, BÖRGERMANN J, KAPPERT U, BREUER M, BÖNING A, URSULESCU A, et al. Off-pump versus onpump coronary-artery bypass grafting in elderly patients. $\mathrm{N}$ Engl J Med 2013; 368: 1189-98.

26. CHIKWE J, LEE T, ITAGAKI S, ADAMS D, EGOROVA N. LONG-TERM Outcomes After Off-Pump Versus On-Pump Coronary Artery Bypass Grafting by Experienced Surgeons. J Am Coll Cardiol 2018; 72: 1478-86.

27. GONZÁLEZ R, REYES R, STOCKINS A, SEGUEL E, JADUE A, ALARCÓN E. Cirugía Coronaria: Resultados Inmediatos y Alejados de la Cirugía de Revascularización Miocárdica en Enfermedad Coronaria. Rev Med Chil2018; 146 : 1395-404. 